\title{
Review
}

\section{Structures of vesicular stomatitis virus glycoprotein: membrane fusion revisited}

\author{
S. Roche ${ }^{*++}$, A. A. V. Albertini, J. Lepault, S. Bressanelli and Y. Gaudin* \\ CNRS, UMR2472, INRA, UMR1157, IFR 115, Virologie Moléculaire et Structurale, 91198, Gif sur Yvette \\ (France), e-mail: gaudin@vms.cnrs-gif.fr, Fax: + 33169824308
}

Received 21 November 2007; received after revision 29 January 2008; accepted 30 January 2008 Online First 17 March 2008

\begin{abstract}
Glycoprotein G of the vesicular stomatitis virus (VSV) is involved in receptor recognition at the host cell surface and then, after endocytosis of the virion, triggers membrane fusion via a low $\mathrm{pH}$ induced structural rearrangement. $G$ is an atypical fusion protein, as there is a $\mathrm{pH}$-dependent equilibrium between its pre- and post-fusion conformations. The atomic structures of these two conformations reveal that it is homologous to glycoprotein $\mathrm{gB}$ of herpesviruses and that it combines features of the previously characterized class I and class II fusion proteins.
\end{abstract}

Comparison of the structures of $\mathrm{G}$ pre- and postfusion states shows a dramatic reorganization of the molecule that is reminiscent of that of paramyxovirus fusion protein $\mathrm{F}$. It also allows identification of conserved key residues that constitute $\mathrm{pH}$-sensitive molecular switches. Besides the similarities with other viral fusion machineries, the fusion properties and structures of $\mathrm{G}$ also reveal some striking particularities that invite us to reconsider a few dogmas concerning fusion proteins.

Keywords. Vesicular stomatitis virus, rhabdovirus, paramyxovirus, glycoprotein, membrane fusion, viral entry, conformational change.

\section{Introduction}

To initiate a productive infection, all viruses must translocate their genome across the cell membrane [1]. For enveloped viruses, this step is mediated by virally encoded glycoproteins that promote both receptor recognition and membrane fusion. Both tasks can be achieved by a single or by separate glycoproteins acting in concert. Activation of the fusion capacity involves large structural rearrangements of the fusogenic glycoproteins upon interaction with specific triggers (e.g. low $\mathrm{pH}$ and cellular

* Corresponding author.

+ Present adress: Max Planck Institute of Biochemistry, Am Klopferspitz 18, 82152 Martinsried (Germany) receptors). These conformational changes result in the exposure of a fusion peptide or fusion loops, which then interact with one or both of the participating membranes, resulting in their destabilization and merger [2]. Triggering of the conformational change in the absence of a target membrane leads to inactivation of the fusion properties of the fusogenic glycoprotein.

Experimental data suggest that the membrane fusion pathway is very similar for all the enveloped viruses studied so far whatever the organization of their fusion machinery [3-5] (Fig. 1). It is generally accepted that fusion proceeds via the formation of intermediate stalks that are local lipidic connections between the outer leaflets of the fusing membranes [6]. Radial expansion of the stalk would induce the 


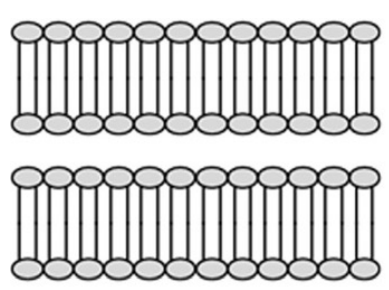

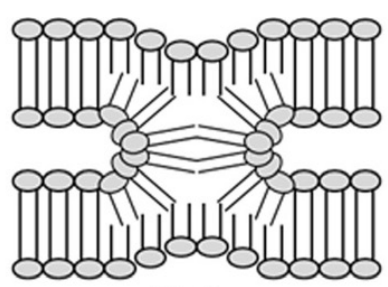

Stalk

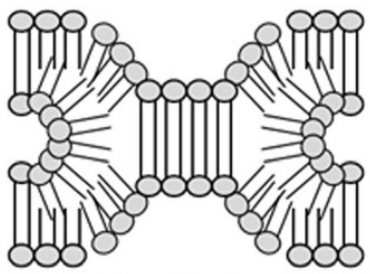

Hemifusion

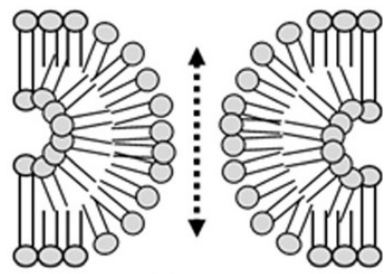

Pore

Figure 1. Stages of membrane fusion according to the stalk-pore model [82].

formation of a transient hemifusion diaphragm (i.e. a local bilayer made by the two initial inner leaflets). Depending on the experimental system, hemifusion may be restricted (i.e. without lipid exchange between the two membranes) or unrestricted (i.e. without any restriction of lipid diffusion). Restriction of lipid flux has been proposed to be due to a ringlike aggregate of fusogenic glycoproteins surrounding the hemifusion diaphragm $[3,4]$. The next step would be the formation of a pore in the fusion diaphragm. The initial pore is small and is often opening and closing repeatedly (the so-called flickering pore) before its enlargement that leads to complete fusion [7].

\section{Rhabdovirus glycoprotein G}

Rhabdoviruses are widespread among a great diversity of organisms (including plants, insects, fishes, mammals, reptiles and crustaceans) [8]. This family includes vesicular stomatitis virus (VSV) as well as significant human pathogens like rabies virus (RV) or Chandipura virus [9]. Rhabdoviruses are enveloped viruses and have in common a bulletlike shape. Their genome is a single RNA molecule of negative polarity. It associates with the nucleoprotein $\mathrm{N}$, the viral polymerase $\mathrm{L}$ and the phosphoprotein $\mathrm{P}$ to form the nucleocapsid. The nucleocapsid is condensed by the matrix protein $\mathrm{M}$ into a tightly coiled helical structure, which is surrounded by a lipid bilayer containing the viral glycoprotein $\mathrm{G}$.

$\mathrm{G}$ forms the spikes that protrude from the viral surface. After cleavage of the aminoterminal signal peptide, the complete mature glycoprotein is about 500 amino acids long (495 for VSV Indiana). The bulk of the mass of $G$ is located outside the viral membrane and constitutes the amino-terminal ectodomain. As this ectodomain is the only outer component of the viruses, it is the target of neutralizing antibodies [1016].

G plays a critical role during the initial steps of virus infection. First, it is responsible for virus attachment to specific receptors. The nature of the receptor remains a matter of debate for both VSV and RV. In the case of
VSV, although phosphatidylserine has been considered to be the viral receptor for a long time [17], recent results indicated that it is not [18]. In the case of RV, many molecules, including gangliosides [19], phospholipids [20], the nicotinic acetylcholine receptor $[21,22]$, neuronal cellular adhesion molecules [23] and the low-affinity nerve growth factor receptor [24], have been proposed to be viral receptors.

After binding, the virions enter the cell by the endocytic pathway. Subsequently, the viral envelope fuses with a cellular membrane within the acidic environment of the endosome [25]. Fusion is triggered by the low $\mathrm{pH}$ of the endosomal compartment and is mediated by the viral glycoprotein. The $\mathrm{pH}$ dependence is very similar from one rhabdovirus to another and the fusion is optimal around $\mathrm{pH} 6$ [26-28]. Preincubation of the virus at low $\mathrm{pH}$ in the absence of a target membrane leads to inhibition of viral fusion. However, this inhibition is reversible, and readjusting the $\mathrm{pH}$ to above 7 leads to the complete recovery of the initial fusion activity. This is the main difference between rhabdoviruses and other viruses fusing at low $\mathrm{pH}$, for which low $\mathrm{pH}$-induced fusion inactivation is irreversible [29].

$\mathrm{G}$ can assume at least three different conformational states having different biochemical and biophysical characteristics $[26,30]$ : the native, prefusion state detected at the viral surface above $\mathrm{pH} 7$; the activated hydrophobic state, which interacts with the target membrane as a first step of the fusion [31]; and the post-fusion conformation, which is antigenically distinct from the native and activated states [32]. There is a $\mathrm{pH}$-dependent equilibrium between the different states of $\mathrm{G}$ that is shifted toward the post-fusion state at low $\mathrm{pH}$ [32]. This indicates that, differently from fusogenic glycoproteins from other viral families, the low-pH induced-conformational change is reversible and thus that the native conformation is not metastable. In fact, the reversibility of the fusogenic low$\mathrm{pH}$-induced conformational change is essential to allow $\mathrm{G}$ to be transported through the acidic compartments of the Golgi apparatus and to recover its native, prefusion state at the viral surface [33]. 


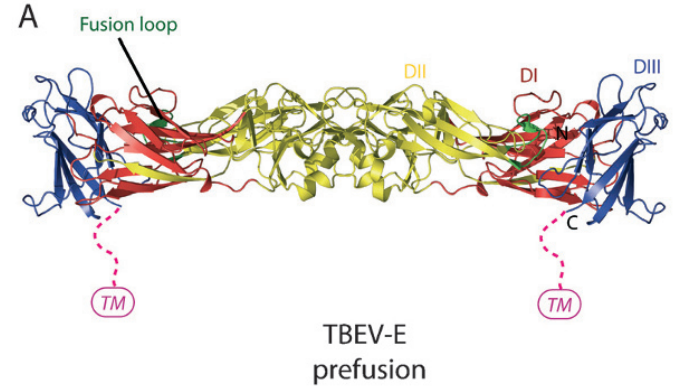

\section{Class I and class II fusion proteins}

Before the structure determination of VSV G, two classes of viral fusion proteins had been identified (Fig. 2, 4). The viral fusion proteins belonging to class I, of which the best-characterized members are the influenza virus hemagglutinin (HA) $[34,35]$ and the fusion protein $(\mathrm{F})$ of the paramyxoviruses $[36,37]$ but which also include fusion proteins from retroviruses [38] and filoviruses [39], are organized in trimers. Each subunit (or protomer) constituting the trimer results from the proteolytic cleavage of a precursor into two fragments. The C-terminal fragment bears at or near its amino-terminal end (i.e. at or near the cleavage site) a hydrophobic fusion peptide, buried at a trimer interface in the prefusion state. In the postfusion conformation, this region refolds as a trimeric coiled coil at the $\mathrm{N}$-terminal end of which are displayed the three fusion peptides and against which are packed, in an antiparallel manner, the segments abutting the transmembrane region (Fig. 4B). The protomer shape is thus an elongated hairpin-like structure with the fusion peptide and the transmembrane domain located at the same end, as expected at the end of the fusion process [40].

The class II fusion proteins contain $\mathrm{E}$ protein of flaviviruses and E1 of alphaviruses [41-43]. They display a molecular architecture completely different from that of class I proteins (Fig. 2). Their fusion peptide is internal, located in a loop between two $\beta$ strands. They are synthesized and folded as a complex with a second viral envelope protein that plays a chaperone role. Proteolytic cleavage of the chaperone primes the fusion protein to trigger membrane merger [44]. In their native conformation (Fig. 2A), they form homo- (flaviviruses) or hetero- (alphaviruses) dimers that are organized in an icosahedral assembly $[42,45]$. They lie flat or nearly flat at the viral surface and their

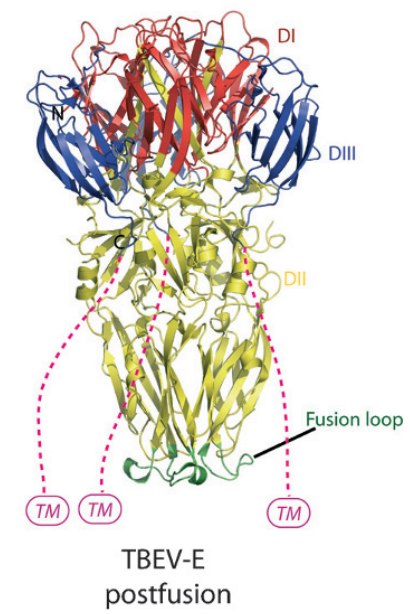

Figure 2. Overall structure of the pre- and post-fusion forms of tick-borne encephalitis virus (TBEV) glycoprotein E, a representative member of class II viral fusion proteins. $(A)$ Ribbon diagram of the dimeric pre-fusion structure (PDB: 1SVB [41]). (B) Ribbon diagram of the trimeric post-fusion structure (PDB: 1URZ [83]). Domains are coloured as in [41]. The location of the fusion loop is indicated. The magenta dotted lines represent the missing part of the ectodomain that is connected to the transmembrane domain. PDB: Protein Data Bank.

fusion loops are buried at a dimer interface. Upon low$\mathrm{pH}$ exposure, dimers dissociate and the protomers reassociate in a trimeric structure $[46,47]$. Similar to the structure of post-fusion class I proteins [48], the fusion loops and the transmembrane domains are then located at the same end of an elongated molecule that is now perpendicular to the membrane $[49,50]$ (Fig. 2B). Thus, even though the structures of class I and class II fusion proteins are unrelated, the mechanisms for refolding share key common features. First, the fusion peptides/loops are exposed and projected toward the top of the glycoprotein, allowing the initial interaction with the target membrane. Second, the folding back of the $\mathrm{C}$-terminal region onto a trimeric $\mathrm{N}$-terminal region leads to the formation of a postfusion protein structure with the outer regions zipped up against the inner trimeric core [2].

For both class I and class II fusion proteins that trigger membrane merger at low $\mathrm{pH}$, the proteolytic cleavage priming the proteins to undergo their low-pH-induced conformational change occurs in the trans-Golgi network or at the host cell surface $[44,51,52]$. This precludes premature activation of the fusion protein in the acidic compartments of the Golgi apparatus. Thus, reversibility of the low-pH-induced fusogenic transition is not necessary for these proteins.

Biochemical, structural and functional properties of rhabdovirus $\mathrm{G}$ suggested that it was distinct from both class I and class II viral fusion proteins that had been already described [29, 53]. Indeed, the $\mathrm{pH}$-dependent equilibrium between the different states of $G$, the absence of predicted $\alpha$-helical coiled-coil motif characteristic of class I viral fusion proteins [40] and the absence of activating cleavage (neither in $\mathrm{G}$ nor in an accompanying protein) strongly suggested that $G$ could define a new category of fusogenic glycoproteins. 


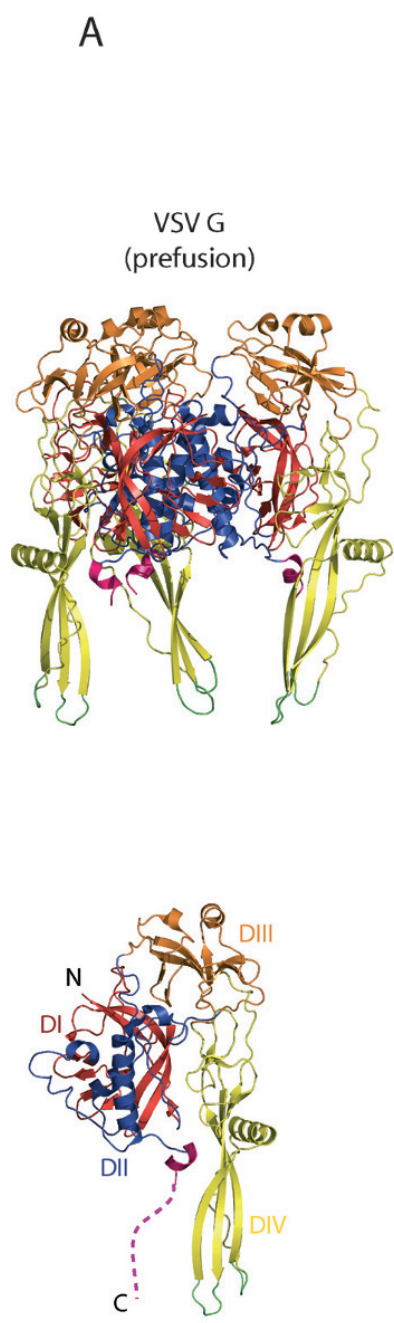

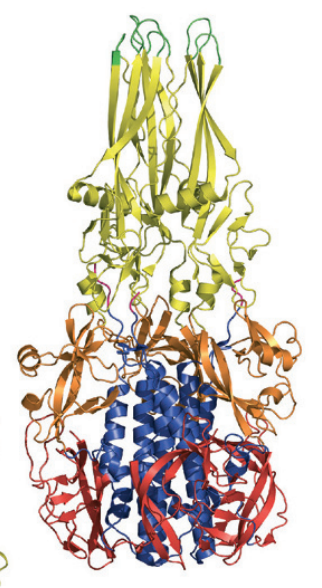

VSV G (postfusion)

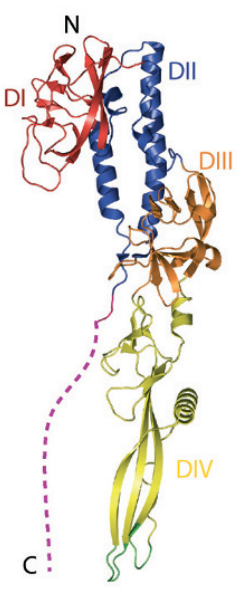

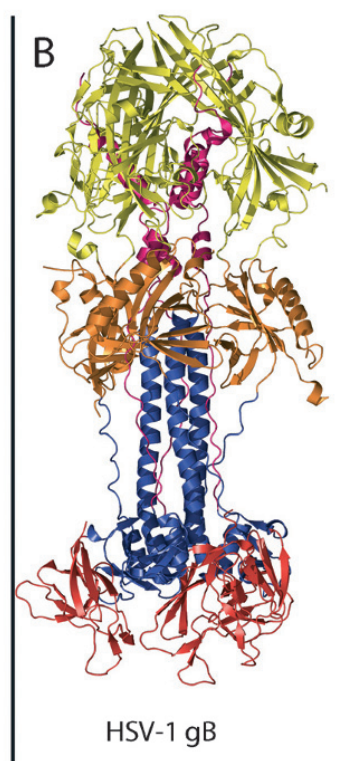

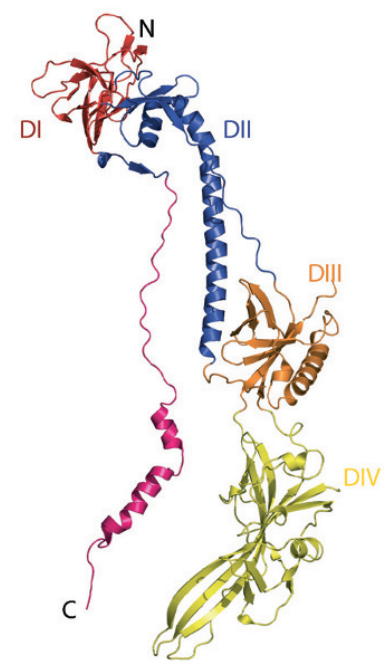

Figure 3. $(A)$ Overall structure of the pre- and post-fusion forms of VSV glycoprotein G. Ribbon diagrams of the pre-fusion structure of G trimer (top left) (PDB: 2J6J [55]); of the post-fusion structure of $\mathrm{G}$ trimer (top right) (PDB: 2CMZ [54]); of the prefusion structure of $\mathrm{G}$ protomer (residues 1-413) (bottom left); and of the post-fusion structure of $\mathrm{G}$ protomer (residues 1-410) (bottom right). $\mathrm{G}$ protein is coloured by domains (domain I: red, domain II: blue, domain III: orange, domain IV: yellow) with the fusion loops in green and the $\mathrm{C}$-terminus in magenta. The protomers are superimposed on their fusion domains (DIV) and the trimers on the rigid blocks made of DI and the invariant part of DII. In the protomer diagrams, the dotted lines represent the missing C-terminal segment of the ectodomain that leads to the transmembrane segment. (B) Overall structure of HSV-1 gB. Ribbon diagrams of $\mathrm{gB}$ trimer (top) and $\mathrm{gB}$ protomer (bottom) (PDB: 2GUM [57]). gB protein is coloured by domains as their homologous counterparts of VSV G.

\section{VSV G structure}

We have recently determined the atomic structures of both the pre- and post-fusion forms of the VSV-G ectodomain, generated by limited proteolysis with thermolysin $\left(\mathrm{G}_{\mathrm{th}}\right.$, aa residues 1-422) [54, 55] (Fig. 3A). The dimensions of the two conformations - consistent with the electron microscopy data obtained on RV G [30,56] - together with the position of the antigenic sites allowed a clear-cut identification of the pre- and post-fusion structures. The structural organization of the two conformations of $G$ is very different from that of other viral fusion proteins described so far. However, amino acid sequence alignment of different $G$ proteins of rhabdoviruses belonging to different genera shows that all these glycoproteins have the same fold.
Four distinct domains of $\mathrm{G}_{\mathrm{th}}$ have been identified: a $\beta$ sheet-rich lateral domain (domain I), a central domain that is involved in the trimerization of the molecule (domain II), a pleckstrin homology domain (domain III) and the fusion domain (domain IV) inserted in a loop of domain III. Major antigenic sites are located in both domains I and III [55].

After the end of the trimerization domain (after amino acid residue 405), there remain 40 amino acids for the polypeptide chain to reach the $\mathrm{G}$ transmembrane domain (Fig. 3A, bottom), but their structural organization is unknown after amino acid residue 413 for the pre-fusion conformation and after amino acid residue 410 for the post-fusion conformation. 


\section{An unexpected homology}

The structure of herpes simplex virus 1 (HSV1, a double-stranded DNA virus) glycoprotein $\mathrm{gB}$ [57] was published at the same time as that of $G_{\text {th }}$ post-fusion state. Comparison of the two structures revealed that their folds are the same and that they have a common evolutionary origin that could not be detected by looking at the amino acid sequences (Fig. 3B). This was completely unexpected and suggests that rhabdoviruses, and most probably all viruses belonging to the Mononegavirales order, have the ability to steal genes from their host (or from another virus during coinfection of a host cell). This might occur when the viral polymerase jumps from the antigenomic template onto an RNA messenger (either of cellular or viral origin) during genomic RNA synthesis. Nevertheless, the exact scenario of $\mathrm{G}$ gene acquisition by the rhabdovirus ancestor will still remain a matter of debate for a long time.

The orientation of the central helix relative to the viral membrane in the determined structure of HSV1 gB suggests that $\mathrm{gB}$ is in its post-fusion conformation. Nevertheless, as the fusion machinery of herpesviruses is much more complex than that of rhabdoviruses (with four glycoproteins that are essential for virus entry) and as its mode of activation is completely different [58], the extent of $\mathrm{gB}$ conformational change cannot be inferred from its homology with VSV G.

\section{The conformational change of VSV G}

Comparison of the pre- and post-fusion structures of VSV $G$ reveals a dramatic reorganization of the molecule (Fig. 3A). During the conformational change, domains I, III and IV retain their tertiary structure. Nevertheless, they undergo large rearrangements in their relative orientation due to secondary structure changes in the hinge regions between the fusion and pleckstrin homology domains and major refolding of the central trimerization domain (Fig. 4C). In fact, the pre- and post-fusion states are related by flipping both the fusion domain and the $\mathrm{C}$ terminal segment relative to a rigid block constituted of the lateral domain and the part of the trimerization domain that retains its structure during the molecule refolding.

Global refolding of $\mathrm{G}$ from pre- to post-fusion conformation exhibits striking similarities to that of class I proteins such as paramyxovirus fusion protein (F) (Fig. 4A) and influenza virus hemagglutinin subunit 2 (HA2) (Fig. 4C) [35, 37]. Particularly, the reversal of the molecule around the rigid block involves the lengthening of the central helices (that form the trimeric central core of the post-fusion conformation, thus displaying the fusion domains through the $\mathrm{PH}$ domains - at their $\mathrm{N}$-termini) and the refolding of the three carboxy-terminal segments into helices that position themselves in the grooves of the central core in an antiparallel manner to form a sixhelix bundle (Fig. 4C). This structural organization is obviously very similar to that of the post-fusion hairpin structure of class I proteins (Fig. 4B), even though, for VSV G, the central helices are not coiled and remain parallel.

\section{Interaction between fusion domains and membranes}

The structural organization of the $\mathrm{G}$ fusion domain resembles that of class II fusion proteins. The main difference is that the membrane interacting motif of the fusion domain is bipartite (as previously proposed for viral haemorrhagic septicaemia virus, another rhabdovirus [27]), made of two loops, and that the loop sequences are not conserved among rhabdoviruses. However, as in class II fusion proteins, these loops always contain aromatic residues and are located at the tip of an elongated three-stranded $\beta$ sheet. Note that in striking contrast to class I and class II viral fusion proteins, the fusion loops are not buried at an oligomeric interface in $\mathrm{G}$ pre-fusion conformation (Fig. 3A). Indeed, these loops are much less hydrophobic than the amino-terminal fusion peptides of class I proteins (even when the three fusion domains of $\mathrm{G}$ are grouped together in the post-fusion conformation). That these loops are indeed an essential part of the membrane interacting motif is consistent with previous mutagenesis work performed on rhabdoviruses [59,60] (Table 1) and has since been confirmed for both VSV G [61] (Table 1) and Herpesviruses $\mathrm{gB}[62,63]$.

Although hydrophobic photolabeling experiments have demonstrated the ability of $\mathrm{G}$ fusion domain to insert into the target membrane as a first step of the fusion process [31, 64], it is clear, from the presence of charged residues in the vicinity of the loops (as in the fusion domain of class II fusion proteins), that any deep penetration inside the membrane is precluded (Fig. 5). Rather, the tryptophans and tyrosines that are found in the fusion loops of all the rhabdoviral $G$ proteins (Fig. 5) act as sticky fingers by positioning themselves at the interface between the fatty acid chains and head group layers of lipids. It is probable that this interfacial interaction involving only a few residues does not create a strong point of anchoring that can be used to pull the target membrane toward the viral one. Rather, we propose that by perturbating the outer leaflet of the target bilayer, it facilitates the 

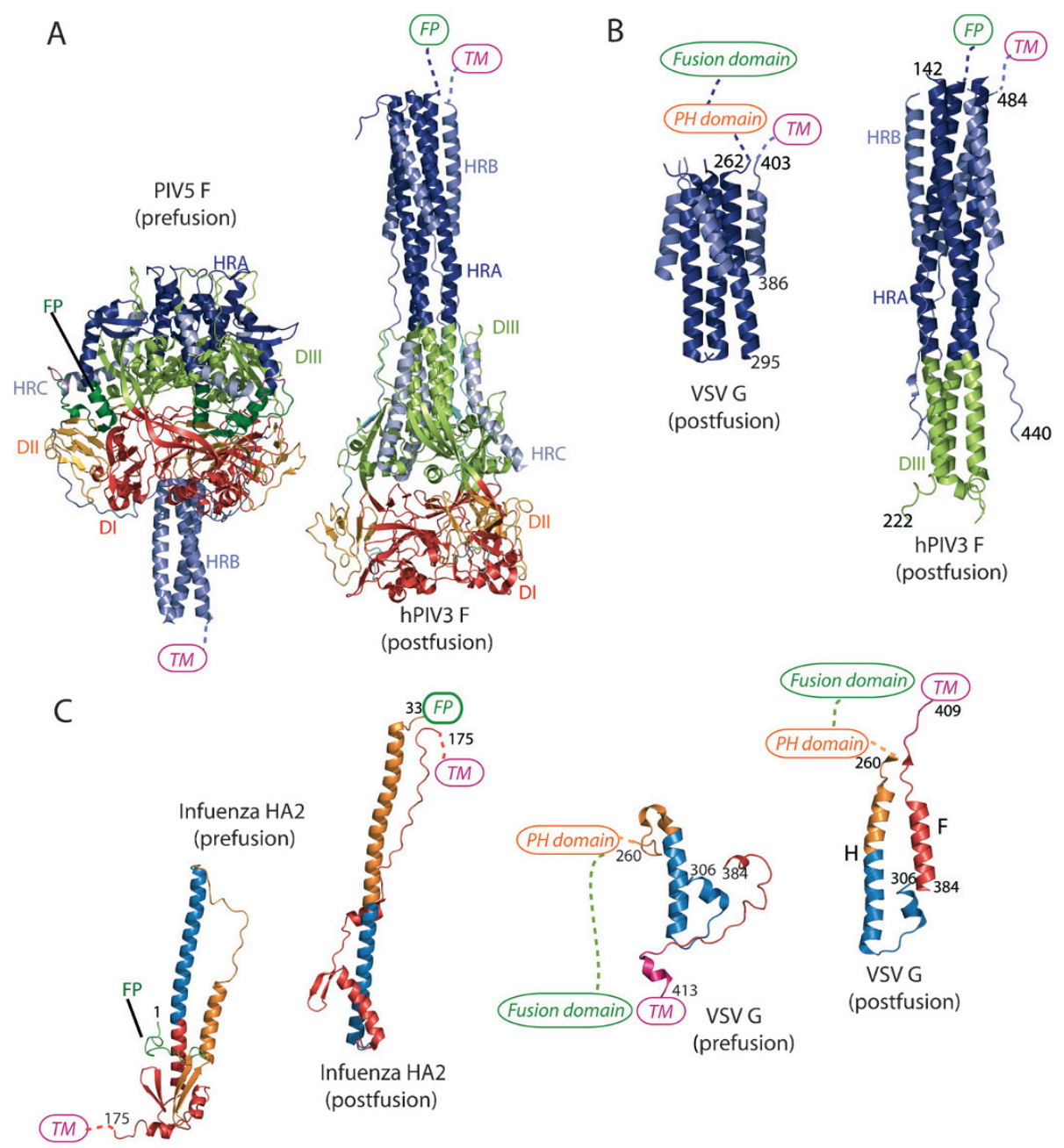

Figure 4. $(A)$ Ribbon diagrams of paramyxovirus $\mathrm{F}$ trimers in the pre-fusion (left) and post-fusion (right) conformations colored by domains (PDB:2B9B,1ZTM [36,37]). Domains and segments are named according to [37]. Note that the two conformations are related by flipping the fusion peptide (FP) and the C-terminal transmembrane domain (TM) relative to a rigid block made of DI and DII. (B) Comparison of the central six-helix bundle of the post-fusion conformation of VSV G with the one of hPIV3 F. hPIV3 F segments are colored according to $(A)$. VSV G helices of the trimerization domain are colored as their hPIV3 F counterpart. The position of the pleckstrin homology (PH), fusion and transmembrane (TM) domains of VSV G are indicated as well as the fusion peptides (FP) and the transmembrane (TM) domains of hPIV3 F. (C) Comparison between the conformational changes of influenza HA2 (left) (PDB: 1HGG, 1HTM $[34,35])$ and the trimerization domain of VSV G. For both polypeptides, unchanged secondary structures are colored in blue; the segment that refolds to form the central helix in the post-fusion conformation is in orange; and the C-terminal part, linked to the transmembrane (TM) domain, that positions itself in the groove of the central core is in red. The position of the pleckstrin homology (PH), fusion and transmembrane (TM) domains of VSV G are indicated as well as the fusion peptide (FP) and the transmembrane (TM) domains of HA2.

formation of pointlike protrusions that have been proposed to be stalk precursors [65].

In $G_{\text {th }}$ pre-fusion conformation, the fusion loops point toward the viral membrane (Fig. 3A). The distance between these loops and the viral membrane essentially depends on the organization of the C-terminal segment of the ectodomain (amino acids residues 414-446) that leads to the TM domain. The structure of this segment is not known, but in the case of RV G, it has been proposed to adopt an $\alpha$-helical conformation [66] having a strong amphipathic signature. Depending on the orientation of this putative helix (either lying flat at the membrane surface or as part of a central trimeric helical bundle perpendicular to the viral membrane), the fusion loops may also interact with the viral membrane when $\mathrm{G}$ is in its pre-fusion conformation.

\section{pH sensitive molecular switches}

Both structures revealed conserved clusters of residues that play the role of $\mathrm{pH}$-sensitive molecular switches. In the pre-fusion conformation [55] there is a 
Table 1. Mutations in rhabdoviral G that affect either the fusion properties or the conformational change of VSV G.

\begin{tabular}{|c|c|c|c|}
\hline Mutation & $\begin{array}{l}\text { Corresponding } \\
\text { residue in VSV G }\end{array}$ & Phenotype & Molecular explanation \\
\hline$V S V$ F2L $\mathrm{L}^{* 1}$ & & $\begin{array}{l}\text { stabilization of the pre-fusion } \\
\text { structure }\end{array}$ & $\begin{array}{l}\text { Residue F2 makes Van der Waals contact with residues I387 and } \\
\text { M391 of the outer helix in the post-fusion hairpin. }\end{array}$ \\
\hline $\begin{array}{l}R V \mathrm{M} 44 \mathrm{~V}^{* 2} \\
R V \mathrm{M} 44 \mathrm{I}^{2} \\
\end{array}$ & $\begin{array}{l}\text { Q42 } \\
\text { Q42 }\end{array}$ & $\begin{array}{l}\text { slower transition from the pre- } \\
\text { to the post-fusion structure }\end{array}$ & $\begin{array}{l}\text { Residue located in the hinge region between the fusion and } \mathrm{PH} \\
\text { domain. }\end{array}$ \\
\hline 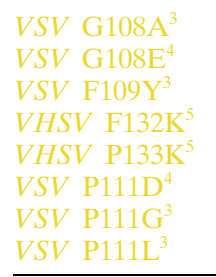 & $\begin{array}{l}\text { F109 } \\
\text { P110 }\end{array}$ & $\begin{array}{l}\text { fusion poorly efficient and/or } \\
\text { only detected at lower } \mathrm{pH}\end{array}$ & $\begin{array}{l}\text { These residues are located in the polyproline helix upstream the } \\
\text { second fusion loop. This structure is involved in lateral interactions } \\
\text { with the neighbouring protomer in the post-fusion conformation. }\end{array}$ \\
\hline $\begin{array}{l}V S V \mathrm{~W} 72 \mathrm{~V}^{6} \\
V S V \mathrm{~W} 72 \mathrm{~A}^{6} \\
V S V \mathrm{Y}^{6} 3 \mathrm{~V}^{6} \\
V S V \mathrm{Y}^{6} \mathrm{~A}^{6} \\
V H S V \mathrm{~F} 100 \mathrm{~K}^{5} \\
V H S V \mathrm{~W} 139 \mathrm{~K}^{5} \\
V S V \mathrm{Y} 116 \mathrm{~V}^{6} \\
V S V \mathrm{Y} 116 \mathrm{~A}^{6} \\
V S V \mathrm{~A} 117 \mathrm{R}^{6} \\
V S V \mathrm{~A} 117 \mathrm{H}^{6} \\
V S V \mathrm{~A} 117 \mathrm{~K}^{4,6} \\
\end{array}$ & $\begin{array}{l}\text { Y73 } \\
\text { Y116 }\end{array}$ & no fusion activity & $\begin{array}{l}\text { Replacement of a hydrophobic residue by a charged one in the fusion } \\
\text { loops or replacement of an aromatic residue by an aliphatic one that is } \\
\text { less prone to destabilize the interface between the fatty acid chains } \\
\text { and head group layers of lipids. }\end{array}$ \\
\hline $\begin{array}{l}R V \mathrm{E} 282 \mathrm{~K}^{* 2} \\
V S V \mathrm{Q} 285 \mathrm{R}^{* 1}\end{array}$ & L283 & $\begin{array}{l}\text { stabilization of the pre-fusion } \\
\text { structure }\end{array}$ & $\begin{array}{l}\text { Residues located in the central helix. Their mutations probably affect } \\
\text { the trimeric interface in one or both conformations. }\end{array}$ \\
\hline $\begin{array}{l}R V \mathrm{~V} 392 \mathrm{G}^{2} \\
R V \mathrm{M} 396 \mathrm{~T}^{2}\end{array}$ & $\begin{array}{l}\mathrm{A} 402 \\
\mathrm{E} 406\end{array}$ & $\begin{array}{l}\text { stabilization of the pre-fusion } \\
\text { structure }\end{array}$ & $\begin{array}{l}\text { Residues located in the hinge region between the trimerization } \\
\text { domain and the C-terminal part of the protomer. }\end{array}$ \\
\hline
\end{tabular}

For RV G and VHSV G mutants, the residue of VSV G that aligns with the mutated position is indicated. The asterisks indicate that the corresponding mutations have been found in a mutant virus. Other mutations were introduced in a plasmid allowing $\mathrm{G}$ expression (and the fusion was assayed by syncytia formation). ${ }^{1}$ From [79]. ${ }^{2}$ From $[80] .{ }^{3}$ From $[81] .{ }^{4}$ From [59]. ${ }^{5}$ From [60]. ${ }^{6}$ From [61].

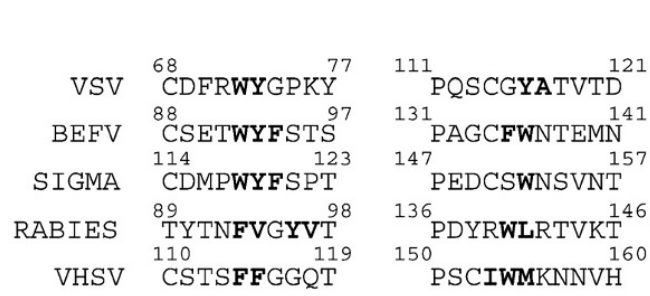

cluster of three histidines: H60 and H162, both located in the fusion domain, and H407, located in the Cterminal part of the protein. Low $\mathrm{pH}$-induced protonation of these residues leads to a cluster of positive charges that might trigger the movement of the fusion domain toward the target membrane. This is consistent with the observed inhibition of VSV fusion by

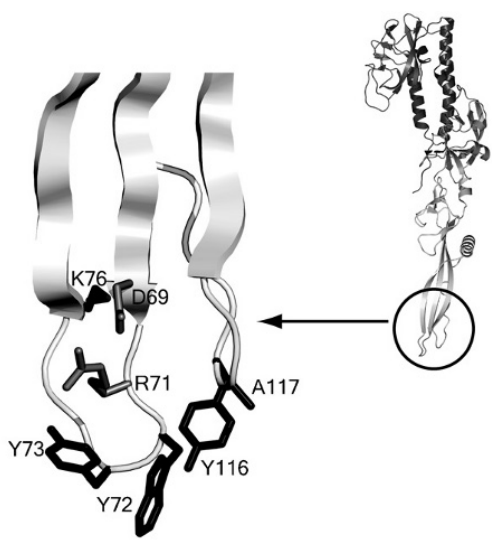

Figure 5. Sequence alignment of the two regions corresponding to the tip of the fusion domain of rhabdoviral $\mathrm{G}$ and close-up view of the tip of VSV G fusion domain showing the two fusion loops. Amino acids that constitute the fusion loops are in bold letters. Hydrophobic residues in the fusion loops are in black. Charged residues that impede deep penetration of the fusion domain in the membrane are in grey. BEFV: Bovine ephemeral fever virus. VHSV: Viral hemorrhagic septicaemia virus. diethylpyrocarbonate, a reagent known to modify specifically histidine residues [67].

Conversely, in the post-fusion state [54] a large number of acidic amino acids are brought close together in the central six-helix bundle. In this protein conformation, they are protonated and form hydrogen bonds. The deprotonation of these residues at higher 


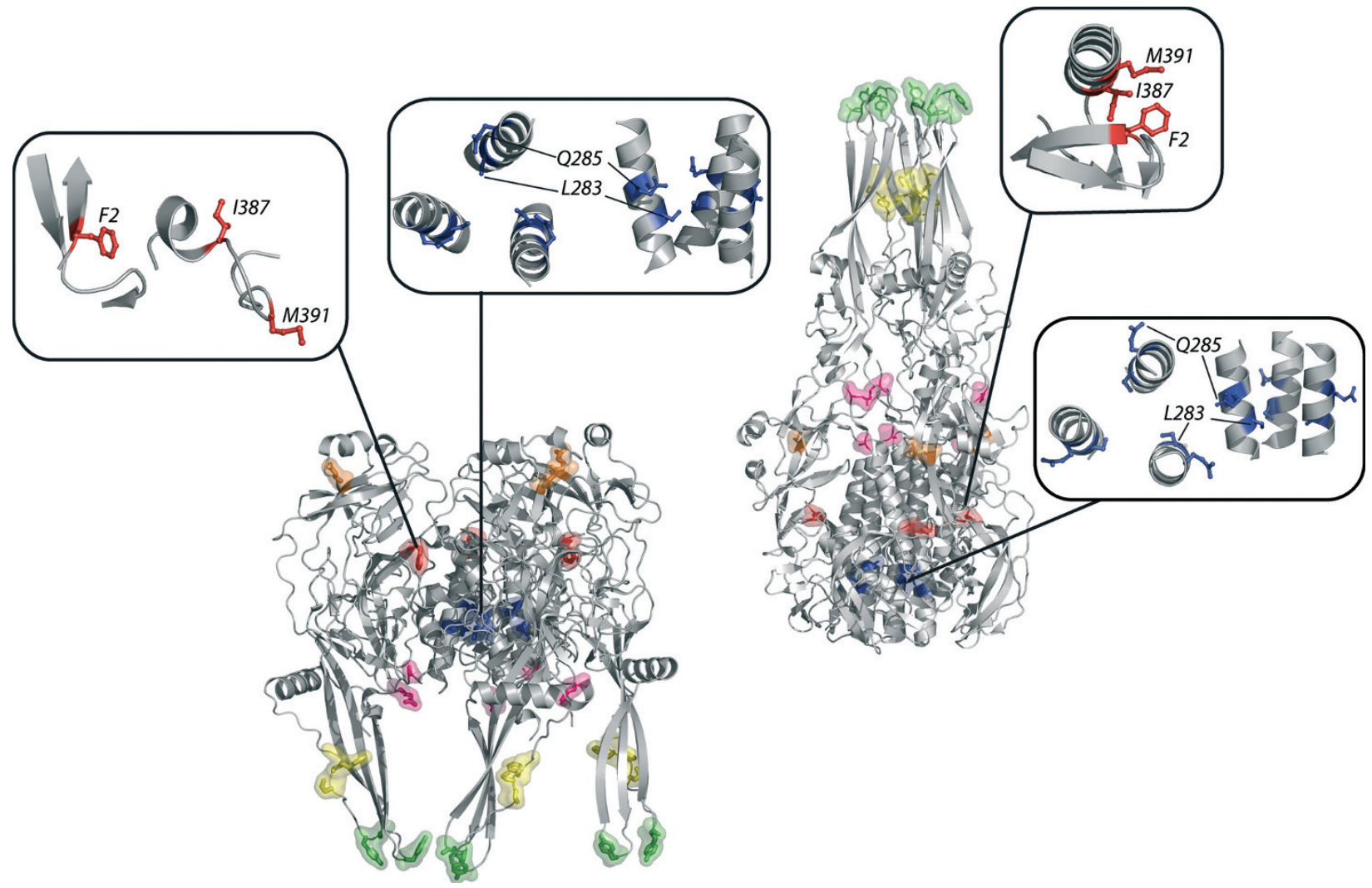

Figure 6. Ribbon diagrams of the pre- and post-fusion conformations of VSV G trimers. The position of the mutations that affect either the conformational change or the fusion properties of $\mathrm{G}$ are colored as indicated in Table 1. Close-up views of the mutations located in the trimeric interface (blue) and of the environment of residue F2 (red) are also shown.

$\mathrm{pH}$ will induce strong repulsive forces that destabilize the trimer and initiate the conformational change back toward the pre-fusion state (in which these residues are solvent-exposed). As the post-fusion form makes the majority of the population up to $\mathrm{pH}$ 6.5 [32], the pKa of these residues in the post-fusion conformation is much higher than their pKa in the prefusion conformation in which they are solvent-exposed. Thus, the post-fusion conformation has a stronger affinity for the protons than the pre-fusion one. This explains the cooperativity of rhabdoviral $\mathrm{G}$ structural transition as a function of $\mathrm{pH}$ [32].

\section{Mutations affecting the structural transition and/or the fusion properties}

A large number of mutations have been described that affect either the structural transition, the fusion properties of rhabdoviral $\mathrm{G}$ or both. The phenotype of some of these mutations most probably results from folding defects often associated with a decrease in transport efficiency. Once this trivial explanation has been discarded, the remaining mutants can be classified in different categories (Table 1). In general, the phenotype of the mutants that affect the stability of the pre-fusion structure can be explained at a molecular level simply by localizing the residue (e.g. those located in hinge regions of the molecule) or by comparing its environment in the pre and post-fusion conformations (Fig. 6). It is also easy to understand why mutations of residues located in the fusion loops abolish G fusion activity (Fig. 6, residues in green). Nevertheless, the fusion phenotype of $G$ having mutations in the segment (Fig. 6, residues in yellow) that adopts a polyproline conformation is not that easy to explain. In the post-fusion conformation, interactions between this segment and $\beta$-strand $d$ of the fusion domain of the neighbouring partner close the bottom of the trimer's internal cavity and keep together the lower part of the fusion domains and thus the fusion loops of the three protomers. Although this underlines the importance of this segment in the regulation of the structural transition and suggests its possible involvement in the oligomerization of intermediates, it is not clear how these mutations affect the efficiency of the fusion process or decrease the optimal fusion $\mathrm{pH}$. 

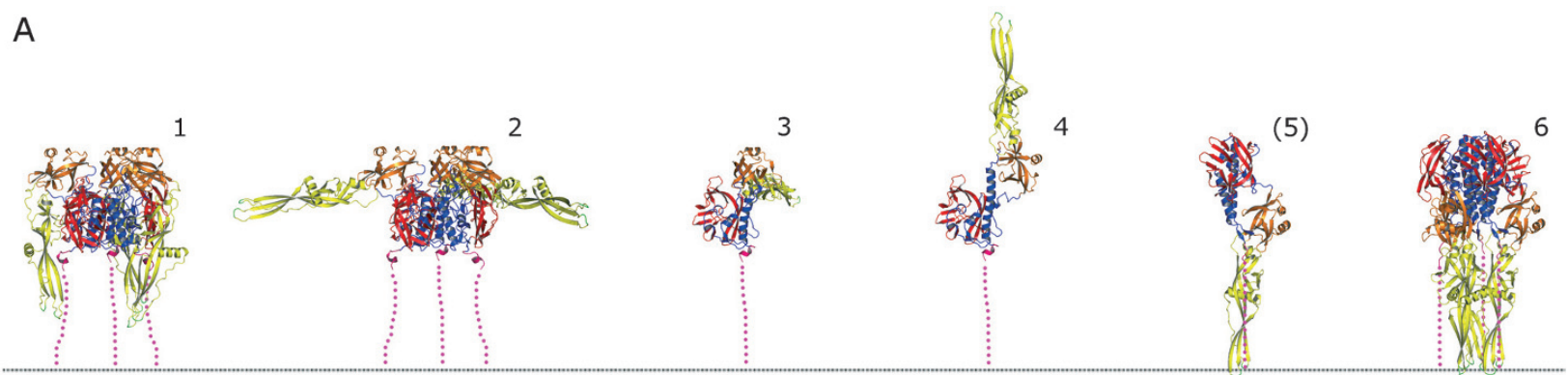

(5)

B

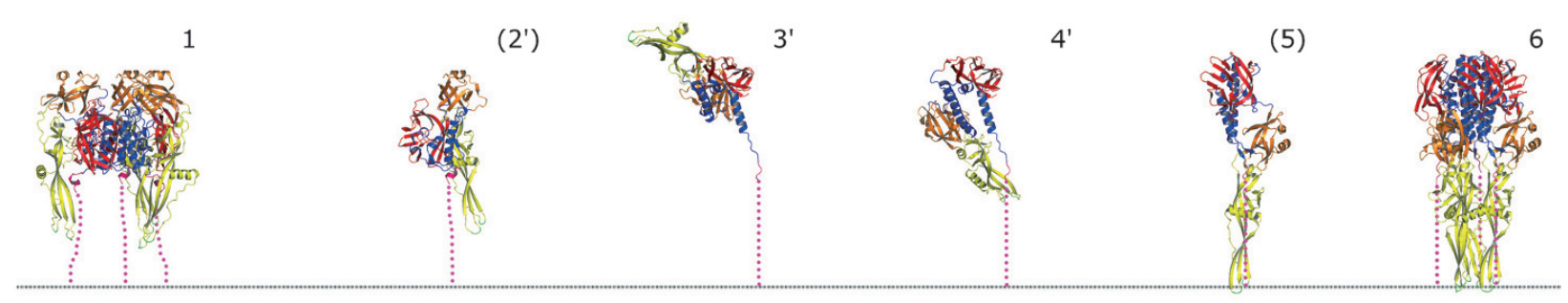

Figure 7. Two possible pathways for the transition from pre- to post-fusion of VSV G at the surface of the viral membrane (depicted by a gray dotted line). Coloured as in Figure 1, with the magenta dotted lines representing the missing part of the ectodomain. 1, pre-fusion trimer, 6, post-fusion trimer, $\left(2^{\prime}\right)$ and (5), pre- and post-fusion protomers, respectively, in parentheses to indicate that these forms are likely transient. The remainder of the forms depicted here are possible intermediates including some but not all of the structural changes. $(A)$ Transition assuming the refolding events occur in the order shown in [55]. 1 to 2 (rotation around the DIV-DIII hinge) can occur without trimer dissociation, but 1 to 4 (adding refolding of the DIII-DII connections) very likely entails monomerization, as the lengthening of the central helix F would lead to clashes at the pre-fusion trimer interface. Refolding of the C-terminus of DII then leads to the post-fusion protomer (5). This last transition likely occurs at the same time as or even after trimer formation, as both events bury acidic amino acids in interfaces (Fig. 3 of [54]). (B) Transition assuming refolding of the C-terminus of DII into helix H (shown in red in Fig. 4C) occurs first (1 to $\left.3^{\prime}\right)$. This implies trimer dissociation [shown as $\left(2^{\circ}\right)$ ], as this segment makes almost all the contacts with the neighbouring protomer. Note that, as for 4, 3' projects the fusion loops away from the viral membrane. $4^{\prime}$, putative intermediate adding the refolding of the DII-DIII and DIII-DIV connections. Finally, $4^{\prime}$ to (5) and 6 is achieved by the packing of helix H against DI (forming the hydrophobic cluster I387-M391F2 shown in Fig. 6) and DII (bringing D393 and E276 into hydrogen-bonding distance, Fig. 3A of [54]).

\section{Intermediates on the fusion pathway}

One intermediate on the fusion pathway exposes the fusion domain at the top of the molecule. This allows the interaction of the fusion loops with the target membrane but is also responsible for the low $\mathrm{pH}$ induced viral aggregation. For rabies virus, this intermediate activated state can be stabilized at low temperature and $\mathrm{pH} 6.4[30,31]$. EM data [30] and antibody binding [32] suggest that the structure of this state is not that different from the native state, raising some questions about the structure of this intermediate (Fig. 7).

Although, for $\mathrm{G}_{\mathrm{th}}$ in solution, it seems possible to go from the pre-fusion trimeric form to its post-fusion counterpart without dissociation and without breaking the threefold symmetry, this seems difficult at the viral surface when the conformational change occurs in the absence of a target membrane (Fig. 7). As there are large differences between the trimeric interfaces of the pre and post-fusion conformations [55], a possible scheme for the pre- to post-fusion transition is that it goes through a transient monomer (as it is the case for class II fusion proteins). This view is also consistent with previous results showing that the prefusion trimer monomerizes after detergent solubilization [68] and that there is an equilibrium between monomers and oligomers in solution [69]. Two plausible pathways are depicted in Figure 7. The first one assumes that the refolding of the molecule implies the initial rotation of the fusion domain (rotation around the DIV-DIII hinge), whereas the second postulates that the refolding of the C-terminus of DII into helix $\mathrm{H}$ (shown in red in Fig. 4C) occurs first.

\section{Cooperativity between a large number of glycoproteins}

The activation energy of the fusion process has been estimated to be in the range of $40 \mathrm{kcal} / \mathrm{mol}[26,70,71]$, most of which is required by enlargement of the initial fusion pore. For class I and class II fusion proteins, it has been proposed that the energy released during the irreversible fusogenic transition is used to achieve the energetically expensive membrane-fusion reaction 


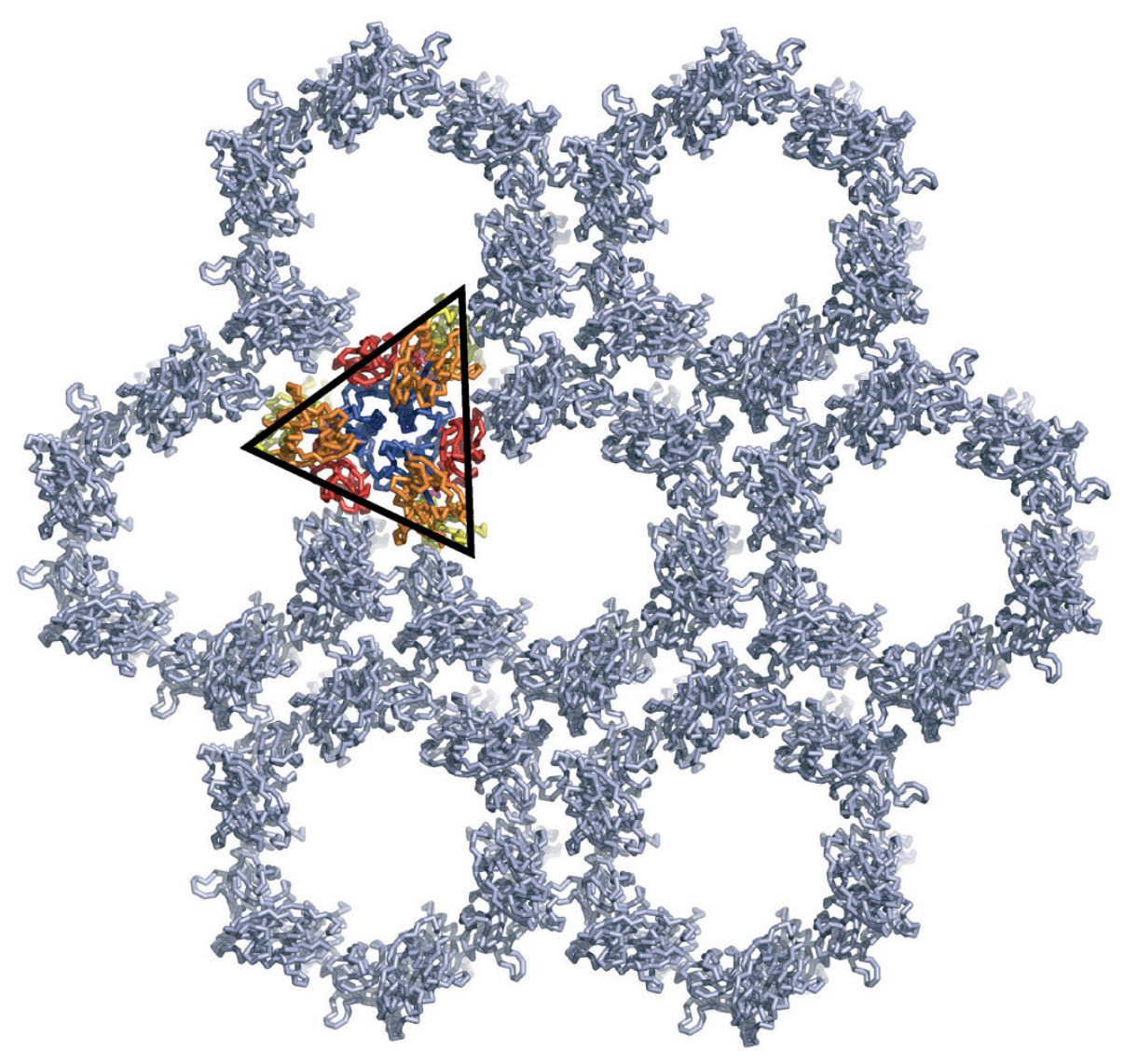

Figure 8. Hexagonal lattice in the crystals of the pre-fusion form of VSV G. One trimer of $\mathrm{G}$ (viewed looking down toward the membrane) is coloured by domain (as in Fig. 3).
$[50,72,73]$, and experimental data suggest that in the case of HIV a single env glycoprotein trimer is sufficient for fusion [74]. Nevertheless, work on many viruses suggests that a large number of spikes are involved during the fusion process.

Data on influenza virus indicate that HA surface density is important for fusion [75] and that its decrease arrests fusion at the hemifusion stage [3] A ringlike organization of HA would also explain the restriction to lipid diffusion observed downstream of stalk formation [3]. Finally, it has been demonstrated that HA molecules located outside the direct contact zone between fusing membranes also contribute to the fusion activity [76].

For class II proteins, different networks of interactions between the glycoproteins have been described. They include the native icosahedral organization of the prefusion dimer covering the entire virus [42,45], the hexagonal lattice of the post-fusion form at the surface of a liposomal lipid bilayer [77] and a putative volcano-shaped ring of five post-fusion trimers [49]. Nevertheless, the exact role of all these networks and complexes remains to be determined.

In the case of rhabdoviruses, the $\mathrm{pH}$-dependent equilibrium between pre- and post-fusion conformations of $\mathrm{G}$ implies that the energy that is released during the structural transition of one trimer cannot be sufficient to overcome the energetic barrier and drive the fusion reaction. Indeed, the minimal number of spikes involved in the formation of an RV fusion complex has been estimated to be about 15 [32]. Interestingly, the p6 lattice organization found in the crystal of the pre-fusion form [55] (Fig. 8) is similar to the local hexagonal lattice observed at the surface of some RV mutants affected in the kinetics of their structural transition when briefly incubated under suboptimal fusion conditions. As for class II proteins, the role of a hexagon (made up of $6 \mathrm{G}$ trimers) or even of the whole network remains to be determined.

\section{Conclusions and perspectives}

The determination of the pre- and post-fusion structures of VSV G is a key step in our understanding of the molecular mechanisms of rhabdovirus viral fusion. Clearly, G combines features of both class I and class II fusion proteins. It also has some striking particularities (e.g. exposition of the fusion loops in the prefusion conformation and reversibility of the conformational change) that invite us to reconsider dogma on membrane fusion and that have to be taken in 
consideration to have a global understanding of virusinduced membrane fusion.

As for other viral fusion machineries, many questions remain unanswered. First, the transmembrane domain is absent in the structures. However, it is known that it plays an important role at the late stages of the fusion process as mutations of transmembrane glycin residues block fusion at a hemifusion stage [78]. Second, the structures of intermediate conformations of G, how they cooperate and how they interact with and deform the viral and target membranes are still very elusive. Understanding the structures of fusion intermediates (made of full-length fusion proteins in association with membranes) is now a major challenge in the field.

Acknowledgements. We acknowledge support from the CNRS and INRA, the CNRS program Physique et Chimie du Vivant, the INRA animal health division program Les virus des animaux et leurs interactions avec la cellule, the MENRT program ACI blanche and the ANR program.

1 Smith, A. E. and Helenius, A. (2004) How viruses enter animal cells. Science 304, 237-242.

2 Weissenhorn, W., Hinz, A. and Gaudin, Y. (2007) Virus membrane fusion. FEBS Lett. 581, 2150-2155.

3 Chernomordik, L. V., Frolov, V. A., Leikina, E., Bronk, P. and Zimmerberg, J. (1998) The pathway of membrane fusion catalyzed by influenza hemagglutinin: restriction of lipids, hemifusion, and lipidic fusion pore formation. J. Cell Biol. 140, $1369-1382$.

4 Gaudin, Y. (2000) Rabies virus-induced membrane fusion pathway. J. Cell Biol. 150, 601-12.

5 Zaitseva, E., Mittal, A., Griffin, D. E. and Chernomordik, L. V. (2005) Class II fusion protein of alphaviruses drives membrane fusion through the same pathway as class I proteins. J. Cell Biol. 169, 167-177.

6 Chernomordik, L. V. and Kozlov, M. M. (2005) Membrane hemifusion: crossing a chasm in two leaps. Cell 123, 375-382.

7 Spruce, A. E., Iwata, A., White, J. M. and Almers, W. (1989) Patch clamp studies of single cell-fusion events mediated by a viral fusion protein. Nature 342, 555-258.

8 Rose, J. K. and Whitt, M. A. (2001) Rhabdoviridae: the viruses and their replication. Fields (4th ed.), 1221-1224.

9 Rao, B. L., Basu, A., Wairagkar, N. S., Gore, M. M., Arankalle, V. A., Thakare, J. P., Jadi, R. S., Rao, K. A. and Mishra, A. C. (2004) A large outbreak of acute encephalitis with high fatality rate in children in Andhra Pradesh, India, in 2003, associated with Chandipura virus. Lancet 364, 869-874.

10 Lefrancois, L. and Lyles, D. S. (1983) Antigenic determinants of vesicular stomatitis virus: analysis with antigenic variants. J. Immunol. 130, 394-398.

11 Vandepol, S. B., Lefrancois, L. and Holland, J. J. (1986) Sequences of the major antibody binding epitopes of the Indiana serotype of vesicular stomatitis virus. Virology 148, $312-325$.

12 Luo, L. H., Li, Y., Snyder, R. M. and Wagner, R. R. (1988) Point mutations in glycoprotein gene of vesicular stomatitis virus (New Jersey serotype) selected by resistance to neutralization by epitope-specific monoclonal antibodies. Virology 163, 341 348.

13 Benmansour, A., Leblois, H., Coulon, P., Tuffereau, C., Gaudin, Y., Flamand, A. and Lafay, F. (1991) Antigenicity of rabies virus glycoprotein. J. Virol. 65, 4198-4203.
14 Prehaud, C., Coulon, P., LaFay, F., Thiers, C. and Flamand, A. (1988) Antigenic site II of the rabies virus glycoprotein: structure and role in viral virulence. J. Virol. 62, 1-7.

15 Seif, I., Coulon, P., Rollin, P. E. and Flamand, A. (1985) Rabies virulence: effect on pathogenicity and sequence characterization of rabies virus mutations affecting antigenic site III of the glycoprotein. J. Virol. 53, 926-934.

16 Flamand, A., Raux, H., Gaudin, Y. and Ruigrok, R. W. (1993) Mechanisms of rabies virus neutralization. Virology 194, 302313.

17 Schlegel, R., Tralka, T. S., Willingham, M. C. and Pastan, I. (1983) Inhibition of VSV binding and infectivity by phosphatidylserine: is phosphatidylserine a VSV-binding site? Cell 32, 639-646.

18 Coil, D. A. and Miller, A. D. (2004) Phosphatidylserine is not the cell surface receptor for vesicular stomatitis virus. J. Virol. 78, 10920-10926.

19 Superti, F., Hauttecoeur, B., Morelec, M. J., Goldoni, P., Bizzini, B. and Tsiang, H. (1986) Involvement of gangliosides in rabies virus infection. J. Gen. Virol. 67, 47-56.

20 Superti, F., Seganti, L., Tsiang, H. and Orsi, N. (1984) Role of phospholipids in rhabdovirus attachment to CER cells. Brief report. Arch. Virol. 81, 321-328.

21 Lentz, T. L., Burrage, T. G., Smith, A. L., Crick, J. and Tignor, G. H. (1982) Is the acetylcholine receptor a rabies virus receptor? Science 215, 182-184.

22 Gastka, M., Horvath, J. and Lentz, T. L. (1996) Rabies virus binding to the nicotinic acetylcholine receptor alpha subunit demonstrated by virus overlay protein binding assay. J. Gen. Virol. 77 (Pt 10), 2437-2440.

23 Thoulouze, M. I., Lafage, M., Schachner, M., Hartmann, U., Cremer, H. and Lafon, M. (1998) The neural cell adhesion molecule is a receptor for rabies virus. J. Virol. 72, 7181-7190.

24 Tuffereau, C., Benejean, J., Blondel, D., Kieffer, B. and Flamand, A. (1998) Low-affinity nerve-growth factor receptor (P75NTR) can serve as a receptor for rabies virus. EMBO J. 17, $7250-7259$

25 Le Blanc, I., Luyet, P. P., Pons, V., Ferguson, C., Emans, N., Petiot, A., Mayran, N., Demaurex, N., Faure, J., Sadoul, R. et al. (2005) Endosome-to-cytosol transport of viral nucleocapsids. Nat. Cell. Biol. 7, 653-664.

26 Clague, M. J., Schoch, C., Zech, L. and Blumenthal, R. (1990) Gating kinetics of $\mathrm{pH}$-activated membrane fusion of vesicular stomatitis virus with cells: stopped-flow measurements by dequenching of octadecylrhodamine fluorescence. Biochemistry 29, 1303-1308.

27 Gaudin, Y., de Kinkelin, P. and Benmansour, A. (1999) Mutations in the glycoprotein of viral haemorrhagic septicaemia virus that affect virulence for fish and the $\mathrm{pH}$ threshold for membrane fusion. J. Gen. Virol. 80, 1221-1229.

28 Roche, S. and Gaudin, Y. (2004) Evidence that rabies virus forms different kinds of fusion machines with different $\mathrm{pH}$ thresholds for fusion. J. Virol. 78, 8746-8752.

29 Gaudin, Y. (2000) Reversibility in fusion protein conformational changes. The intriguing case of rhabdovirus-induced membrane fusion. Subcell. Biochem. 34, 379-408.

30 Gaudin, Y., Ruigrok, R. W., Knossow, M. and Flamand, A. (1993) Low-pH conformational changes of rabies virus glycoprotein and their role in membrane fusion. J. Virol. 67, 136572.

31 Durrer, P., Gaudin, Y., Ruigrok, R. W., Graf, R. and Brunner, J. (1995) Photolabeling identifies a putative fusion domain in the envelope glycoprotein of rabies and vesicular stomatitis viruses. J. Biol. Chem. 270, 17575-17581.

32 Roche, S. and Gaudin, Y. (2002) Characterization of the equilibrium between the native and fusion-inactive conformation of rabies virus glycoprotein indicates that the fusion complex is made of several trimers. Virology 297, 128-135.

33 Gaudin, Y., Tuffereau, C., Durrer, P., Flamand, A. and Ruigrok, R. W. (1995) Biological function of the low-pH, fusion-inactive conformation of rabies virus glycoprotein $(\mathrm{G})$ : 
$\mathrm{G}$ is transported in a fusion-inactive state-like conformation. $\mathrm{J}$ Virol. 69, 5528-5534.

34 Wilson, I. A., Skehel, J. J. and Wiley, D. C. (1981) Structure of the haemagglutinin membrane glycoprotein of influenza virus at 3 A resolution. Nature $289,366-373$.

35 Bullough, P. A., Hughson, F. M., Skehel, J. J. and Wiley, D. C. (1994) Structure of influenza haemagglutinin at the $\mathrm{pH}$ of membrane fusion. Nature 371, 37-43.

36 Yin, H. S., Paterson, R. G., Wen, X., Lamb, R. A. and Jardetzky, T. S. (2005) Structure of the uncleaved ectodomain of the paramyxovirus (hPIV3) fusion protein. Proc. Natl. Acad. Sci. USA 102, 9288-93.

37 Yin, H. S., Wen, X., Paterson, R. G., Lamb, R. A. and Jardetzky, T. S. (2006) Structure of the parainfluenza virus 5 F protein in its metastable, pre-fusion conformation. Nature 439, $38-44$.

38 Weissenhorn, W., Calder, L. J., Dessen, A., Laue, T., Skehel, J. J. and Wiley, D. C. (1997) Assembly of a rod-shaped chimera of a trimeric GCN4 zipper and the HIV-1 gp41 ectodomain expressed in Escherichia coli. Proc. Natl. Acad. Sci. USA 94, 6065-6069.

39 Weissenhorn, W., Calder, L. J., Wharton, S. A., Skehel, J. J. and Wiley, D. C. (1998) The central structural feature of the membrane fusion protein subunit from the Ebola virus glycoprotein is a long triple-stranded coiled coil. Proc. Natl. Acad. Sci. USA 95, 6032-6036.

40 Skehel, J. J. and Wiley, D. C. (1998) Coiled coils in both intracellular vesicle and viral membrane fusion. Cell 95, 871874.

41 Rey, F. A., Heinz, F. X., Mandl, C., Kunz, C. and Harrison, S. C. (1995) The envelope glycoprotein from tick-borne encephalitis virus at 2 A resolution. Nature 375, 291-298.

42 Lescar, J., Roussel, A., Wien, M. W., Navaza, J., Fuller, S. D., Wengler, G., Wengler, G. and Rey, F. A. (2001) The Fusion glycoprotein shell of Semliki Forest virus: an icosahedra assembly primed for fusogenic activation at endosomal $\mathrm{pH}$. Cell 105, 137-148.

43 Modis, Y., Ogata, S., Clements, D. and Harrison, S. C. (2003) A ligand-binding pocket in the dengue virus envelope glycoprotein. Proc. Natl. Acad. Sci. USA 100, 6986-6991.

44 Allison, S. L., Stadler, K., Mandl, C. W., Kunz, C. and Heinz, F. X. (1995) Synthesis and secretion of recombinant tick-borne encephalitis virus protein $\mathrm{E}$ in soluble and particulate form. $\mathrm{J}$ Virol. 69, 5816-5820.

45 Kuhn, R. J., Zhang, W., Rossmann, M. G., Pletnev, S. V., Corver, J., Lenches, E., Jones, C. T., Mukhopadhyay, S., Chipman, P. R., Strauss, E. G., Baker, T. S. and Strauss, J. H. (2002) Structure of dengue virus: implications for flavivirus organization, maturation, and fusion. Cell 108, 717-725.

46 Wahlberg, J. M. and Garoff, H. (1992) Membrane fusion process of Semliki Forest virus. I: Low $\mathrm{pH}$-induced rearrangement in spike protein quaternary structure precedes virus penetration into cells. J. Cell Biol. 116, 339-348.

47 Stiasny, K., Allison, S. L., Marchler-Bauer, A., Kunz, C. and Heinz, F. X. (1996) Structural requirements for low-pHinduced rearrangements in the envelope glycoprotein of tickborne encephalitis virus. J. Virol. 70, 8142-8147.

48 Kielian, M. and Rey, F. A. (2006) Virus membrane-fusion proteins: more than one way to make a hairpin. Nat. Rev. Microbiol. 4, 67-76.

49 Gibbons, D. L., Vaney, M. C., Roussel, A., Vigouroux, A., Reilly, B., Lepault, J., Kielian, M. and Rey, F. A. (2004) Conformational change and protein-protein interactions of the fusion protein of Semliki Forest virus. Nature 427, 320-325.

50 Modis, Y., Ogata, S., Clements, D. and Harrison, S. C. (2004) Structure of the dengue virus envelope protein after membrane fusion. Nature 427, 313-319.

51 Lobigs, M. and Garoff, H. (1990) Fusion function of the Semliki Forest virus spike is activated by proteolytic cleavage of the envelope glycoprotein precursor p62. J. Virol. 64, 1233-1240.

52 Schafer, W., Stroh, A., Berghofer, S., Seiler, J., Vey, M., Kruse, M. L., Kern, H. F., Klenk, H. D. and Garten, W. (1995) Two independent targeting signals in the cytoplasmic domain determine trans-Golgi network localization and endosomal trafficking of the proprotein convertase furin. EMBO J. 14, 2424-2435.

53 Da Poian, A. T., Carneiro, F. A. and Stauffer, F. (2005) Viral membrane fusion: is glycoprotein $\mathrm{G}$ of rhabdoviruses a representative of a new class of viral fusion proteins? Braz. J. Med. Biol. Res. 38, 813-823.

54 Roche, S., Bressanelli, S., Rey, F. A. and Gaudin, Y. (2006) Crystal structure of the low-pH form of the vesicular stomatitis virus glycoprotein G. Science 313, 187-191.

55 Roche, S., Rey, F. A., Gaudin, Y. and Bressanelli, S. (2007) Structure of the pre-fusion form of the vesicular stomatitis virus glycoprotein g. Science 315, 843-848.

56 Gaudin, Y., Ruigrok, R. W., Tuffereau, C., Knossow, M. and Flamand, A. (1992) Rabies virus glycoprotein is a trimer. Virology 187, 627-632.

57 Heldwein, E. E., Lou, H., Bender, F. C., Cohen, G. H., Eisenberg, R. J. and Harrison, S. C. (2006) Crystal structure of glycoprotein B from herpes simplex virus 1 . Science $313,217-$ 220.

58 Spear, P. G. and Longnecker, R. (2003) Herpesvirus entry: an update. J. Virol. 77, 10179-10185.

59 Fredericksen, B. L. and Whitt, M. A. (1995) Vesicular stomatitis virus glycoprotein mutations that affect membrane fusion activity and abolish virus infectivity. J. Virol. 69, 14351443.

60 Rocha, A., Ruiz, S., Tafalla, C. and Coll, J. M. (2004) Conformation- and fusion-defective mutations in the hypothetical phospholipid-binding and fusion peptides of viral hemorrhagic septicemia salmonid rhabdovirus protein G. J. Virol. 78, 9115-9122.

61 Sun, X., Belouzard, S. and Whittaker, G. R. (2007) Molecular architecture of the bipartite fusion loops of vesicular stomatitis virus glycoprotein G, a class III viral fusion protein. J. Biol. Chem. [Epub ahead of print]

62 Backovic, M., Jardetzky, T. S. and Longnecker, R. (2007) Hydrophobic residues that form putative fusion loops of Epstein-Barr virus glycoprotein B are critical for fusion activity. J. Virol. 81, 9596-9600.

63 Hannah, B. P., Heldwein, E. E., Bender, F. C., Cohen, G. H. and Eisenberg, R. J. (2007) Mutational evidence of internal fusion loops in herpes simplex virus glycoprotein B. J. Virol. 81, 48584865.

64 Pak, C. C., Puri, A. and Blumenthal, R. (1997) Conformational changes and fusion activity of vesicular stomatitis virus glycoprotein: [125I]iodonaphthyl azide photolabeling studies in biological membranes. Biochemistry 36, 8890-8896.

65 Efrat, A., Chernomordik, L. V. and Kozlov, M. M. (2007) Pointlike protrusion as a prestalk intermediate in membrane fusion pathway. Biophys J 92, L61-63.

66 Maillard, A., Domanski, M., Brunet, P., Chaffotte, A., Guittet, E. and Gaudin, Y. (2003) Spectroscopic characterization of two peptides derived from the stem of rabies virus glycoprotein. Virus Res. 93, 151-158.

67 Carneiro, F. A., Stauffer, F., Lima, C. S., Juliano, M. A., Juliano, L. and Da Poian, A. T. (2003) Membrane fusion induced by vesicular stomatitis virus depends on histidine protonation. J. Biol. Chem. 278, 13789-13794.

68 Doms, R. W., Keller, D. S., Helenius, A. and Balch, W. E. (1987) Role for adenosine triphosphate in regulating the assembly and transport of vesicular stomatitis virus $G$ protein trimers. J. Cell Biol. 105, 1957-1969.

69 Lyles, D. S., Varela, V. A. and Parce, J. W. (1990) Dynamic nature of the quaternary structure of the vesicular stomatitis virus envelope glycoprotein. Biochemistry 29, 2442-2449.

70 Lee, J. and Lentz, B. R. (1998) Secretory and viral fusion may share mechanistic events with fusion between curved lipid bilayers. Proc. Natl. Acad. Sci. USA 95, 9274-9279.

71 Kuzmin, P. I., Zimmerberg, J., Chizmadzhev, Y. A. and Cohen, F. S. (2001) A quantitative model for membrane fusion based 
on low-energy intermediates. Proc. Natl. Acad. Sci. USA 98, $7235-7240$

72 Ruigrok, R. W., Martin, S. R., Wharton, S. A., Skehel, J. J., Bayley, P. M. and Wiley, D. C. (1986) Conformational changes in the hemagglutinin of influenza virus which accompany heatinduced fusion of virus with liposomes. Virology 155, 484-497.

73 Carr, C. M., Chaudhry, C. and Kim, P. S. (1997) Influenza hemagglutinin is spring-loaded by a metastable native conformation. Proc. Natl. Acad. Sci. USA 94, 14306-14313.

74 Yang, X., Kurteva, S., Ren, X., Lee, S. and Sodroski, J. (2005) Stoichiometry of envelope glycoprotein trimers in the entry of human immunodeficiency virus type 1 . J. Virol. 79, 1213212147.

75 Danieli, T., Pelletier, S. L., Henis, Y. I. and White, J. M. (1996) Membrane fusion mediated by the influenza virus hemagglutinin requires the concerted action of at least three hemagglutinin trimers. J. Cell Biol. 133, 559-569.

76 Leikina, E., Mittal, A., Cho, M. S., Melikov, K., Kozlov, M. M. and Chernomordik, L. V. (2004) Influenza hemagglutinins outside of the contact zone are necessary for fusion pore expansion. J. Biol. Chem. 279, 26526-26532.

77 Gibbons, D. L., Erk, I., Reilly, B., Navaza, J., Kielian, M., Rey, F. A. and Lepault, J. (2003) Visualization of the targetmembrane-inserted fusion protein of Semliki Forest virus by combined electron microscopy and crystallography. Cell 114, 573-583.

78 Cleverley, D. Z. and Lenard, J. (1998) The transmembrane domain in viral fusion: essential role for a conserved glycine residue in vesicular stomatitis virus $G$ protein. Proc. Natl. Acad. Sci. USA 95, 3425-3430.

79 Martinez, I. and Wertz, G. W. (2005) Biological differences between vesicular stomatitis virus Indiana and New Jersey serotype glycoproteins: identification of amino acid residues modulating $\mathrm{pH}$-dependent infectivity. J. Virol. 79, 3578-3585.

80 Gaudin, Y., Raux, H., Flamand, A. and Ruigrok, R. W. (1996) Identification of amino acids controlling the low-pH-induced conformational change of rabies virus glycoprotein. J. Virol. 70, $7371-7378$

81 Zhang, L. and Ghosh, H. P. (1994) Characterization of the putative fusogenic domain in vesicular stomatitis virus glycoprotein G. J. Virol. 68, 2186-2193.

82 Chernomordik, L., Kozlov, M. M. and Zimmerberg, J. (1995) Lipids in biological membrane fusion. J. Membr. Biol. 146, 114.

83 Bressanelli, S., Stiasny, K., Allison, S. L., Stura, E. A., Duquerroy, S., Lescar, J., Heinz, F. X. and Rey, F. A. (2004) Structure of a flavivirus envelope glycoprotein in its low- $\mathrm{pH}$ induced membrane fusion conformation. EMBO J. 23, $728-$ 738.

\section{To access this journal online: http://www.birkhauser.ch/CMLS}

